Research article

\title{
Analysis of medicinal and economic important plant species of Hollongapar Gibbon wildlife sanctuary, Assam, northeast India
}

\author{
Moumita Sarkar and Ashalata Devi*
}

Department of Environmental Science, Tezpur University, Tezpur-784028, Sonitpur, Assam, India

*Corresponding Author: ashalatadevi12@gmail.com

[Accepted: 23 December 2017]

\begin{abstract}
An investigation has been made to recognise the medicinal and economic potential of plant species occurred in the semi-evergreen forest of Hollongapar Gibbon wildlife sanctuary, Assam using semi-structured questionnaire. In the present study, the importance of plant species recorded in this semi-evergreen forest is analysed and assessed in terms of their medicinal and economic values. A total of 157 plant species belonging to 136 genera and 78 families were having medicinal and economic values. These include 69 trees (55 genera and 39 families), 17 shrubs (15 genera and 14 families), 58 herbs (57 genera and 37 families), 5 lianas ( 5 genera and 5 families) and 8 bamboo/cane/palm (5 genera and 2 families). The study revealed $78 \%$ of plant species were having significant values either in terms of medicinal or economic and both which make the plant diversity of the sanctuary a vital source for resource supply. Majority of the recorded medicinal plants were used for the treatment of some common health problems such as fever, cough, cold, skin diseases, jaundice, dysentery, etc. Non Timber Forest products consist of wild edible vegetables, resins, gums, fire woods, fodder, wild edible fruits, bamboo, canes, etc.
\end{abstract}

Keywords: Plant diversity - Economic plants - Medicinal plants - Anthropogenic activities Conservation.

[Cite as: Sarkar M \& Devi A (2017) Analysis of medicinal and economic important plant species of Hollongapar Gibbon wildlife sanctuary, Assam, northeast India. Tropical Plant Research 4(3): 486-495]

\section{INTRODUCTION}

Tropical forests are rich centres of species diversity having high productivity which results in a great supply of resources to mankind. The services provided by tropical forests are numerous and taken as granted. Contribution to human welfare with their distinctive medicinally and economically important plants is worth mentioning. In general, forests provide a variety of resources which may be of direct or indirect use to human life and regarded as a vital source of livelihood. The fundamental social, ethical, cultural and economic values of humans have directly or indirectly revolved around biological resources since the earliest date of historical record (Ramesh 2003, Pandey \& Pandey 2016). People have traditionally and substantially depended upon the resources in the wild for their sustenance (Gadgil 1989, Mehra et al. 2014, Bajpai et al. 2016).

The northeast region of India is a centre of rich biodiversity in the Indian sub-continent with a significant proportion of flora and fauna having medicinal and economic values. Assam a state of northeast India is a constituent unit of the Eastern Himalayan Biodiversity Region which is one of the three biodiversity hotspots in the country. In Assam, there are 23 protected areas represented by 5 national parks and 18 wildlife sanctuaries. The total area under national parks and wildlife sanctuaries in 2012 is $3925 \mathrm{Km}^{2}$. contributing $5 \%$ of state's geographical area (PCCF 2012). Several studies have been undertaken in different protected areas likewise in the Hollongapar Gibbon wildlife sanctuary, Assam. However, most of the study undertaken in the sanctuary was found to primarily focus on the primate species (Tilson 1979, Chetry 2001, Hazarika \& Gupta 2005, Chetry et al. 2007, Chetia \& Kalita 2012). Bujarbarua (2002) carried out an ecological study of the sanctuary. A study on Poaceae family of the sanctuary was done by Bujarbarua \& Sarma (2006) where a total number of 37 species belonging to 22 genera were collected. Verma et al. (2012) enumerated liverwort and hornwort flora of the sanctuary. An analysis of tree species diversity, population dynamics and assessment of their regeneration 
pattern was studied by Sarkar \& Devi (2014a) while, Borah \& Devi (2014) carried out the ecological study on a critically endangered tree species (Vatica lanceaefolia B1.) in the sanctuary. However, there is no record of assessment of the potential value of plant species diversity occurred in the sanctuary in terms of medicinal and economic importance and their subsequent utilization. Therefore, an attempt has been made to investigate the potential value of plant species diversity occurred in Hollongapar Gibbon wildlife sanctuary, Assam.

\section{MATERIALS AND METHODS}

The Hollongapar Gibbon wildlife sanctuary (HGWLS), popularly known as Gibbon wildlife sanctuary, is an

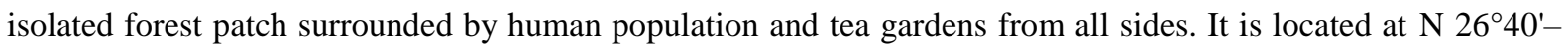
$26^{\circ} 45^{\prime}$ and $\mathrm{E} 94^{\circ} 20^{\prime}-94^{\circ} 25^{\prime}$ at an altitudinal range of $100-120 \mathrm{~m}$ amsl, in Mariani, Jorhat district of upper Assam. It covers an area of $20.98 \mathrm{Km}^{2}$ and the sanctuary has been divided into five compartments. The HGWLS falls under "North East Biogeographic Zone (9)" and "N.E. Brahmaputra Valley Biogeographic Province (9A)"as per Rodgers \& Panwar (1988) and the forest type is "Eastern Alluvial Secondary Semi-Evergreen Forest (1/2/2B/2S2)" under Moist Tropical Forests of India (Champion \& Seth 1968). The climate in the region may be classified as sub-tropical humid type (monsoonal climate) having four seasons in a year: pre-monsoon (March to May), monsoon (June to September), post-monsoon (October to November) and winter (December to February). The location of this sanctuary and accessibility by the local inhabitants of the fringe area for resource utilization has made this site important for ecological studies.

Importance of plant species occurred in HGWLS was investigated by collecting relevant data using informal semi-structured questionnaire from the people in terms of medicinal and economic importance. The local people inhabited in and around the sanctuary were interview through informal conversation and any information on uses of the plant in different proposes was recorded using the questionnaire and other relevant information were also noted. Prior Information Consent (PIC) was taken before the conversation/interview. Literature like 'Flora of Assam' (Kanjilal \& Bor 2005) was also consulted for any relevant information to be incorporated.

\section{RESULTS}

A total of 100 individuals/villagers inhabited in and around the sanctuary, belonging to the age group of 3080, were interviewed for collecting the information on the importance of plant species. Analysing the collected data it was found that several plant species having medicinal and economic values were collected from the sanctuary by the villagers for their subsistence. A total of 157 species belonging to 136 genera and 78 families were found having ethnobotanical values which includes 69 trees (55 genera and 39 families), 5 lianas (5 genera and 5 families), 17 shrubs ( 15 genera and 14 families), 58 herbs ( 57 genera and 37 families), and 8 species under bamboo, cane and palm (5 genera and 2 families). Detail of these recorded ethnobotanical important plant species in terms of medicinal and economic values is given in table 1. It was estimated that $78 \%$ of plant species were having certain significant values either medicinal or economic or both which make the plant diversity of the sanctuary a vital source of resource supply to the nearby inhabitants. Out of the 157 species, 52 species were exclusively used for a medicinal purpose which belonged to 51 genera and 34 families. Out of these 52 species, 3 species were trees ( 2 genera and 2 families), 9 species were shrubs (9 genera and 8 families) and 40 species were herbs (40 genera and 25 families). These plants like Dillenia indica, Macaranga denticulata, Terminalia chebula, Clerodendrum infortunatum, Phlogacanthus thyrsiformis, Cheilocostus speciosus, Cissampelos pareira, Commelina benghalensis, Paederia scandens, Phyllanthus fraternus, etc. are widely used for the treatment of some common health problems such as fever, cough, cold, skin diseases, jaundice, dysentery, etc. While, 36 species belonging to 33 genera and 23 families were having economic values. Among the 36 species, 21 species were trees ( 20 genera and 19 families), 1 species was shrub, 3 species were herbs ( 3 genera and 3 families), 4 species were lianas ( 4 genera and 4 families), 3 species were bamboo ( 3 genera and 1 family), 3 species were cane ( 1 genus and 1 family) and 1 species was palm. 15 species were found to be used exclusively for timber. These plants are important and found to be used extensively as wild edible vegetables, resins, gums, fire woods, fodder, wild edible fruits, etc. by the local people inhabited in and around the sanctuary to meet their economic subsistence.

\section{DISCUSSION AND CONCLUSION}

The sanctuary harbours a rich diversity of medicinal and economic important plants which adds the potential value of the sanctuary. The commercially important plant species enumerated in the study area supports 
Table 1. List of medicinal and economically important plant species recorded in Hollongapar Gibbon wildlife sanctuary.

\begin{tabular}{|c|c|c|c|c|c|c|}
\hline \multirow{2}{*}{$\frac{\text { S.N. }}{\text { Tree }}$} & \multirow[t]{2}{*}{ Name of Species } & \multirow[t]{2}{*}{ Family } & \multirow[t]{2}{*}{ Ver. name } & \multicolumn{2}{|c|}{ Importance } & \multirow[t]{2}{*}{ Uses } \\
\hline & & & & & & \\
\hline 1 & Aglaia spectabilis (Miq.) S.S. Jain \& Bennet & Meliaceae & Amari & - & $\mathrm{E}$ & Timber \\
\hline 2 & Ailanthus integrifolia Lam. & Simaroubaceae & Borpaat & - & E & Timber \\
\hline 3 & Albizia lebbek (L.) Benth. & Mimosaceae & Shirish & - & E & Timber \\
\hline 4 & Alseodaphne petiolaris Hook. f. & Lauraceae & Gojua & M & - & Jaundice \\
\hline 5 & Alstonia scholaris (L.) R. Br. & Apocynaceae & Sotiona & M & E & Timber \\
\hline 6 & Altingia excelsa Noronha & Altingiaceae & Jutuli & M & $\mathrm{E}$ & Headache, allergy, boils; timber \\
\hline 7 & Aquilaria malaccensis Lam. & Thymelaeaceae & Agaru & M & $\mathrm{E}$ & Skin diseases; resin, oil, highly scented wood \\
\hline 8 & Artocarpus chaplasha Roxb. & Moraceae & Samkothal & M & $\mathrm{E}$ & Diarrhoea, sores; timber, fruit edible \\
\hline 9 & Artocarpus lakoocha Roxb. & Moraceae & Bohot & M & $\mathrm{E}$ & Skin diseases; fruit and flower head edible,dye \\
\hline 10 & Baccaurea ramiflora Lour. & Phyllanthaceae & Leteku & M & $\mathrm{E}$ & Stomach ache, toothache; fruit edible, fish farming \\
\hline 11 & Balakata baccata (Roxb.) Esser & Euphorbiaceae & Seleng & - & E & Timber, wood \\
\hline 12 & Barringtonia acutangula (L.) Gaertn. & Lecythidaceae & Paani amara & M & $\mathrm{E}$ & $\begin{array}{l}\text { Diarrhoea, syphilis, leprosy; timber, bark for } \\
\text { intoxicating fish }\end{array}$ \\
\hline 13 & Canarium bengalense Roxb. & Burseraceae & Dhuna & M & $\mathrm{E}$ & Rashes, snake bite; resin, air purifier \\
\hline 14 & Carallia brachiata (Lour.) Merr. & Rhizophoraceae & Maahithekera & M & $\mathrm{E}$ & Stomach disorder; Timber, fruit edible \\
\hline 15 & Castanopsis indica (Roxb. ex Lindl.) A.DC. & Fagaceae & Hingori & - & $\mathrm{E}$ & Leaves used for cigarettes, fruit edible, timber \\
\hline 16 & Castanopsis tribuloides (Sm.) A.DC. & Fagaceae & Phoolhingori & M & E & Cough, goitre, indigestion; Timber, fruit edible \\
\hline 17 & Chukrasia tabularis A.Juss. & Meliaceae & Bogapoma & M & $\mathrm{E}$ & Stomach ache, diarrhoea, dysentery; timber \\
\hline 18 & Cinnamomum glaucescens (Nees) Hand.-Mazz. & Lauraceae & Gonsoroi & - & $\mathrm{E}$ & Timber \\
\hline 19 & Cyathea gigantea (Wall. ex Hook.) Holtt. & Cyatheaceae & Tree fern & M & $\mathrm{E}$ & Anti-inflammatory; stem edible \\
\hline 20 & Dillenia indica $\mathrm{L}$. & Dilleniaceae & Outenga & M & $\mathrm{E}$ & $\begin{array}{l}\text { Stomach ache, jaundice, diarrhoea, dysentery, } \\
\text { dandruff; timber, fruit edible }\end{array}$ \\
\hline 21 & Dipterocarpus retusus $\mathrm{Bl}$ & Dipterocarpaceae & Holong & - & $\mathrm{E}$ & Timber \\
\hline 22 & Drimycarpus racemosus (Roxb.) Hook.f. ex Marchand. & Anacardiaceae & Aamsia & - & $\mathrm{E}$ & Timber \\
\hline 23 & Duabanga grandiflora (DC.) Walp. & Lythraceae & Khakan & - & $\mathrm{E}$ & Timber \\
\hline 24 & Dysoxylum gotadhora (Buch.-Ham.) Mabb. & Meliaceae & Bandardima & M & $\mathrm{E}$ & Diarrhoea, dysentery; timber \\
\hline 25 & Elaeocarpus serratus $\mathrm{L}$. & Elaeocarpaceae & Rudraksh & - & E & Cultural and religious use \\
\hline 26 & Eurya acuminata DC. & Pentaphylacaceae & Murmura & $\mathrm{M}$ & $\mathrm{E}$ & Diarrhoea; fuelwood, dye \\
\hline 27 & Evodia meliaefolia Benth. & Rutaceae & Maiphak & - & E & Timber \\
\hline
\end{tabular}




\begin{tabular}{|c|c|c|c|c|c|c|}
\hline 28 & Ficus benghalensis L. & Moraceae & Borgoch & M & $\mathrm{E}$ & $\begin{array}{l}\text { Rheumatism, diarrhoea, dysentery, diabetes; timber, } \\
\text { fodder }\end{array}$ \\
\hline 29 & Ficus benjamina $\mathrm{L}$. & Moraceae & Jorigoch & M & - & Stomach disorder \\
\hline 30 & Ficus fistulosa Reinw. ex Bl. & Moraceae & Kathiadimoru & M & $\mathrm{E}$ & Post-natal treatment, diaphoretic; firewood \\
\hline 31 & Ficus lamponga Miq. & Moraceae & Dimoru & M & - & Jaundice \\
\hline 32 & Ficus racemosa $\mathrm{L}$. & Moraceae & Jagya Dimoru & M & $\mathrm{E}$ & $\begin{array}{l}\text { Diabetes, liver disorders, diarrhoea, urinary diseases; } \\
\text { fruit edible }\end{array}$ \\
\hline 33 & Ficus religiosa $\mathrm{L}$. & Moraceae & Ahot & M & $\mathrm{E}$ & $\begin{array}{l}\text { Dysentery, fever, scabies, piles, skin diseases, } \\
\text { gonorrhoea; religious, cultural, Timber, fodder, fruit } \\
\text { edible }\end{array}$ \\
\hline 34 & Garcinia morella (Gaertn.) Desr. & Clusiaceae & Kujithekera & M & $\mathrm{E}$ & Diarrhoea, leprosy, ulcers; resin, oil, dye, fruit edible \\
\hline 35 & Garcinia pedunculata Roxb. ex Buch.-Ham. & Clusiaceae & Borthekera & M & $\mathrm{E}$ & Diarrhoea, dysentery, jaundice; fruit edible \\
\hline 36 & Gmelina arborea Roxb. & Lamiaceae & Gamari & M & $\mathrm{E}$ & Stomach disorder; timber, fruit and flower edible \\
\hline 37 & Hydnocarpus kurzii (King) Warb. & Achariaceae & Saalmugura & M & $\mathrm{E}$ & Leprosy; oil \\
\hline 38 & Ilex godajam Coleb. ex Hook.f. & Aquifoliaceae & Haatikerepa & - & $\mathrm{E}$ & Firewood \\
\hline 39 & Kydia calycina Roxb. & Malvaceae & Pisola & M & $\mathrm{E}$ & $\begin{array}{l}\text { Skin diseases, wounds, cuts, boils, veterinary } \\
\text { medicine; timber, fibrous bark }\end{array}$ \\
\hline 40 & Lagerstroemia speciosa (L.) Pers. & Lythraceae & Azar & M & $\mathrm{E}$ & $\begin{array}{l}\text { Diarrhoea, dysentery, jaundice; timber, cultivated for } \\
\text { flowers }\end{array}$ \\
\hline 41 & Litsea monopetala (Roxb.) Pres. & Lauraceae & Sualu & M & $\mathrm{E}$ & Stomach ache; mugasilk worm reared on leaves \\
\hline 42 & Macaranga denticulata (Blume) Müll.Arg. & Euphorbiaceae & Moralia & M & $\mathrm{E}$ & Skin diseases, cuts, wounds; firewood, fodder \\
\hline 43 & Magnolia champaca (L.) Baill. ex Pierre & Magnoliaceae & Titasopa & M & $\mathrm{E}$ & Chronic gastritis, cough, fever; timber \\
\hline 44 & Magnolia hodgsonii (Hook. f. \& Th.) H. Keng & Magnoliaceae & Borhomothuri & M & $\mathrm{E}$ & Tooth and gum; firewood, dye, timber \\
\hline 45 & Magnolia hookeri (Cubitt\& Smith) Raju \& Nayar & Magnoliaceae & Paansopa & - & $\mathrm{E}$ & Timber \\
\hline 46 & Magnolia oblonga (Wall. ex Hook.f. \& Thomson) Figlar & Magnoliaceae & Borsopa & - & $\mathrm{E}$ & Timber \\
\hline 47 & Mallotus nudiflorus (L.) Kulju \& Welzen & Euphorbiaceae & Bhelkar & - & $\mathrm{E}$ & Timber \\
\hline 48 & Mangifera sylvatica Roxb. & Anacardiaceae & Bon-aam & M & $\mathrm{E}$ & $\begin{array}{l}\text { Gastrointestinal disorder; unripe fruit for pickles, } \\
\text { jelly and tarts }\end{array}$ \\
\hline 49 & Mesua ferrea $\mathrm{L}$. & Calophyllaceae & Nahor & M & $\mathrm{E}$ & $\begin{array}{l}\text { Fever, vomiting, urinary tract disorders, migraine; } \\
\text { oil, timber }\end{array}$ \\
\hline 50 & Morinda angustifolia Roxb. & Rubiaceae & Aasugoch & M & $\mathrm{E}$ & Body pain, cough, cracked feet; dye \\
\hline 51 & Neolamarckia cadamba (Roxb.) Bosser & Rubiaceae & Kadam & M & $\mathrm{E}$ & $\begin{array}{l}\text { Blood purifier, antidiuretic, abortifacient; timber, } \\
\text { fruit edible }\end{array}$ \\
\hline 52 & Olea dioica Roxb. & Oleaceae & Poreng & M & $\mathrm{E}$ & Skin diseases, fever; fuelwood, charcoal \\
\hline 53 & Palaquium obovatum (Griff.) Engl. & Sapotaceae & Kotholua & - & $\mathrm{E}$ & Yields an inferior kind of Gutta percha \\
\hline
\end{tabular}


Sarkar \& Devi (2017) 4(3): 486-495

\begin{tabular}{|c|c|c|c|c|c|c|}
\hline 54 & Premna bengalensis $\mathrm{Cl}$. & Lamiaceae & Gohora & - & $\mathrm{E}$ & Timber \\
\hline 55 & Pterospermum acerifolium (L.) Willd. & Malvaceae & Mota-marulia & M & $\mathrm{E}$ & $\begin{array}{l}\text { Glandular swelling of neck and ears; fodder, roofing } \\
\text { material }\end{array}$ \\
\hline 56 & Saurauia roxburghii Wall. & Saurauiaceae & Bonposola & M & $\mathrm{E}$ & Constipation; fruit edible, fodder, country liquor \\
\hline 57 & Spondias mombin L. & Anacardiaceae & Khamolimola & - & $\mathrm{E}$ & Bark chewed as substitute for areca nut, fruit edible \\
\hline 58 & Spondias pinnata (L.f.) Kurz. & Anacardiaceae & Amara & M & $\mathrm{E}$ & Dysentery; fruits and flower buds edible \\
\hline 59 & Sterculia villosa Roxb. & Malvaceae & Udal & M & $\mathrm{E}$ & Dysentery; timber,fibrous bark, seeds edible \\
\hline 60 & Stereospermum chelonoides (L.f.) DC. & Bignoniaceae & Paroli & M & $\mathrm{E}$ & Skin diseases, cough, arthritis; timber \\
\hline 61 & Symplocos ferruginea Roxb. & Symplocaceae & Motabhomloti & - & $\mathrm{E}$ & Fruits used for rosaries \\
\hline 62 & Syzygium kurzii (Duthie) Balakr. & Myrtaceae & Bogijamuk & M & $\mathrm{E}$ & Stomach trouble; timber \\
\hline 63 & Terminalia catappa $\mathrm{L}$. & Combretaceae & Kaathbadam & - & $\mathrm{E}$ & Kernel edible, cultivated for fruits, dye \\
\hline 64 & Terminalia chebula Retz. & Combretaceae & Hilikha & M & $\mathrm{E}$ & $\begin{array}{l}\text { Diarrhoea, dysentery, bleeding gums, conjunctivitis, } \\
\text { appetizer; timber, tanning }\end{array}$ \\
\hline 65 & Terminalia myriocarpa Van Heurck \& Müll. Arg. & Combretaceae & Halakh & M & $\mathrm{E}$ & Urinary disorder; Timber, charcoal \\
\hline 66 & Tetrameles nudiflora $\mathrm{R} . \mathrm{Br}$. & Tetramelaceae & Bheleu & - & $\mathrm{E}$ & Timber \\
\hline 67 & Vatica lanceaefolia $\mathrm{Bl}$. & Dipterocarpaceae & Morsal & M & $\mathrm{E}$ & Dysentery; firewood, resin, oil \\
\hline 68 & Vernonia arborea Buch.-Ham. & Asteraceae & Maskoita & - & $\mathrm{E}$ & Bark chewed as substitute for betel leaf \\
\hline 69 & Walsura robusta Roxb. & Meliaceae & Lali & M & $\mathrm{E}$ & Antibacterial, antioxidant; timber \\
\hline \multicolumn{7}{|c|}{ Shrub } \\
\hline 70 & Clerodendrum glandulosum Lindl. & Lamiaceae & Nephaphu & M & - & High blood pressure, hypertension \\
\hline 71 & Clerodendrum infortunatum $\mathrm{L}$. & Lamiaceae & Dhapatphool & M & $\mathrm{E}$ & $\begin{array}{l}\text { Skin diseases, bee's sting; roots used for fermenting } \\
\text { liquor }\end{array}$ \\
\hline 72 & Clerodendrum japonicum (Thunb.) Sweet & Lamiaceae & Dhapattita & M & $\mathrm{E}$ & Febrile and catarrhal affection; leaves edible \\
\hline 73 & Croton joufra Roxb. & Euphorbiaceae & Mahudi & M & - & Skin diseases, anticancer, antioxidant \\
\hline 74 & Dendrocnide sinuata (Blume) Chew & Urticaceae & Churaat & M & - & Jaundice, urogenital disorder, toothache, dysentery \\
\hline 75 & Grewia multiflora Juss. & Malvaceae & Kukurhuta & M & $\mathrm{E}$ & Dysentery, fruit edible, lac insect reared on this plant \\
\hline 76 & Ixora acuminata Roxb. & Rubiaceae & Borpotia & M & - & Jaundice \\
\hline 77 & Lantana camara L. var. aculeata (L.) Mold. & Verbenaceae & Bon baahaar & M & $\mathrm{E}$ & Tetanus; insect repellent \\
\hline 78 & Leea indica (Burm.f.) Merr. & Vitaceae & Kukurathengia & M & $\mathrm{E}$ & Digestive disorders; fruit edible, dye \\
\hline 79 & Melastoma malabathricum $\mathrm{L}$. & Melastomataceae & Phutuka & M & $\mathrm{E}$ & $\begin{array}{l}\text { Cuts, wounds, tooth and gum diseases; fruit } \\
\text { edible,stem used as toothbrush }\end{array}$ \\
\hline 80 & Mussaenda frondosa $\mathrm{L}$. & Rubiaceae & Chubaiata & M & - & Jaundice, ulcer, fever, wounds, asthma \\
\hline 81 & Pandanus odorifer (Forssk.) Kuntze & Pandanaceae & Keyakothal & M & - & Pneumonia \\
\hline
\end{tabular}




\begin{tabular}{|c|c|c|c|c|c|c|}
\hline 82 & Phlogacanthus thyrsiformis (Roxb. ex Hardw.) Mabb. & Acanthaceae & Titaphool & M & $\mathrm{E}$ & Cough, fever, abdominal pain; flowers edible \\
\hline 83 & Rauvolfia serpentina (L.) Benth. ex Kurz & Apocynaceae & Sarpagandha & M & - & Snake bite, insomnia, stomach ache \\
\hline 84 & Syzygium fruticosum DC. & Myrtaceae & Katahijamuk & M & - & Blood dysentery \\
\hline 85 & Viburnum colebrookeanum Wall. & Caprifoliaceae & Mezenga & M & - & Sores \\
\hline 86 & Ziziphus funiculosa Buch.-Ham. & Rhamnaceae & Bonbogori & - & $\mathrm{E}$ & Fruits edible \\
\hline \multicolumn{7}{|c|}{ Herb } \\
\hline 87 & Abrus precatorius L. & Papilionaceae & Latumoni & M & - & Abortifacient, induce sterility in women \\
\hline 88 & Ageratum conyzoides $\mathrm{L}$. & Asteraceae & Gendheli bon & M & - & Cuts, wounds, jaundice \\
\hline 89 & Alocasia forniculata (Roxb.) Schott. & Araceae & Adoliakochu & M & - & Crack of heels \\
\hline 90 & Alpinia nigra (Gaertn.) Burtt. & Zingiberaceae & Tora/Bogitora & M & - & Bone weakness, irregular menstruation \\
\hline 91 & Begonia roxburghii A. DC. & Begoniaceae & Begonia tenga & M & - & Skin diseases \\
\hline 92 & Cheilocostus speciosus (J.König) C.Specht & Costaceae & Jamlakhuti & M & - & Jaundice, insect bite \\
\hline 93 & Chromolaena odorata (L.) R.M. King \& H. Rob. & Asteraceae & Germany bon & M & - & Cuts, wounds \\
\hline 94 & Chrysopogon aciculatus (Retz.) Trin. & Poaceae & Bonguti & M & - & Arthritis, rheumatism,antidote (sting) \\
\hline 95 & Cissampelos pareira $\mathrm{L}$. & Menispermaceae & Tubukilata & M & & Fever, headache \\
\hline 96 & Commelina benghalensis L. & Commelinaceae & Kanasimolu & M & $\mathrm{E}$ & Snake bite, leprosy, skin inflammations; fodder \\
\hline 97 & Curcuma aromatica Salisb. & Zingiberaceae & Bonhaladi & M & - & Blood purification, constipation \\
\hline 98 & Cuscuta reflexa Roxb. & Convolvulaceae & Raghumala & M & - & $\begin{array}{l}\text { Induce sterility in women, purgative, veterinary } \\
\text { medicine }\end{array}$ \\
\hline 99 & Cynodon dactylon (L.) Pers. & Poaceae & Dubori bon & M & - & Headache, irregular menstruation, tonic \\
\hline 100 & Cyperus rotundus $\mathrm{L}$. & Cyperaceae & Keyabon & M & - & Stomach ache, purgative \\
\hline 101 & Dicranopteris linearis (Burm.f.) Underw. & Gleicheniaceae & Kap-dhekia & M & $\mathrm{E}$ & $\begin{array}{l}\text { Indigestion, asthma, women's sterility, fever, } \\
\text { anticancer; vascular bundles from the stalks woven to } \\
\text { make products }\end{array}$ \\
\hline 102 & Dioscorea bulbifera $\mathrm{L}$. & Dioscoreaceae & Aalulata & M & - & Dysentery, indigestion \\
\hline 103 & Diplazium esculentum (Retz.) Sw. & Woodsiaceae & Dhekiasak & M & $\mathrm{E}$ & Constipation, young fronds edible \\
\hline 104 & Eleusine indica Gaertn. & Poaceae & Bobosabon & M & - & $\begin{array}{l}\text { Post partum aidto mother, effective in fracture of } \\
\text { bones of hen and duck }\end{array}$ \\
\hline 105 & Emilia sonchifolia DC. & Asteraceae & Bonkopahua & M & - & Eye inflammation, febrifuge \\
\hline 106 & Eragrostis unioloides Nees ex Steud. & Poaceae & - & M & - & Asthma, wounds \\
\hline 107 & Evolvulus nummularius L. & Convolvulaceae & - & M & - & Burns, cuts, wounds \\
\hline 108 & Floscopa scandens Lour. & Commelinaceae & - & M & - & Sore eyes, fractured bone \\
\hline 109 & Gonostegia hirta (Blume ex Hassk.) Miq. & Urticaceae & Sialkotahi & - & $\mathrm{E}$ & Roots are used as hair wash, stem and leaves edible \\
\hline
\end{tabular}


Sarkar \& Devi (2017) 4(3): 486-495

\begin{tabular}{|c|c|c|c|c|c|c|}
\hline 110 & Hodgsonia macrocarpa (Bl.) Cogn. & Cucurbitaceae & Thebou & $\mathrm{M}$ & $\mathrm{E}$ & $\begin{array}{l}\text { Antifertility; seed kernels edible, silk worms are fed } \\
\text { on the leaves }\end{array}$ \\
\hline 111 & Homalomena aromatica (Spreng.) Schott & Araceae & Gankochu & $\mathrm{M}$ & $\mathrm{E}$ & Liver and kidney disorder; essential oil \\
\hline 112 & Impatiens balsamina $\mathrm{L}$. & Balsaminaceae & Demdeuka & $\mathrm{M}$ & - & Fever, sterility \\
\hline 113 & Lasia spinosa Thw. & Araceae & Chengmora & M & - & Piles, sore throat, irregular menstruation \\
\hline 114 & Leuca splukenetii Spreng. & Lamiaceae & Durun & M & $\mathrm{E}$ & $\begin{array}{l}\text { Piles, tonsillitis, stomach trouble, nasal haemorrhage; } \\
\text { young shoots edible }\end{array}$ \\
\hline 115 & Lindernia crustacea (L.) F. Muell. & Linderniaceae & Kaachidoria & $\mathrm{M}$ & - & Diabetes, dysentery, boils, ringworm infection \\
\hline 116 & Ludwigia octovalvis (Jacq.) P.H. Raven & Onagraceae & Longbon & M & - & Fever, dysentery, jaundice, cancer \\
\hline 117 & Lygodium microphyllum (Cav.) R.Br. & Schizaeaceae & Kopoudhekia & $\mathrm{M}$ & $\mathrm{E}$ & $\begin{array}{l}\text { Dysentery, skin diseases; basket making and plaiting } \\
\text { from old stems, young stems edible }\end{array}$ \\
\hline 118 & Mikania scandens (L.) Willd. & Asteraceae & - & $\mathrm{M}$ & - & Antifungal \\
\hline 119 & Millettia pachycarpa Benth. & Papilionaceae & Bokolbihlata & M & $\mathrm{E}$ & Skin infection; fish poison \\
\hline 120 & Mimosa pudica $\mathrm{L}$. & Mimosaceae & Nilajibon & M & - & Toothache, skin diseases, piles, boils \\
\hline 121 & Murdannia nudiflora (L.) Brenan & Commelinaceae & - & M & - & Asthma, leprosy, piles \\
\hline 122 & Oxalis debilis var. corymbosa (DC.) Lour. & Oxalidaceae & Bor-tengechi & M & - & Dysentery \\
\hline 123 & Paederia scandens (Lour.) Merr. & Rubiaceae & Bhedailata & $\mathrm{M}$ & - & Diarrhoea, dysentery, rheumatism \\
\hline 124 & Persicaria barbata (L.) H. Hara & Polygonaceae & - & $\mathrm{M}$ & - & Ulcer, purgative, tonic \\
\hline 125 & Phrynium pubinerve $\mathrm{B} 1$. & Marantaceae & Koupat & M & - & Skin diseases, boils, leprosy \\
\hline 126 & Phyllanthus fraternus Webster & Phyllanthaceae & Bon aamlokhi & $\mathrm{M}$ & - & Dysentery, urinary trouble \\
\hline 127 & Piper longum $\mathrm{L}$. & Piperaceae & Pipoli & M & $\mathrm{E}$ & Cough, cold, loss of appetite; condiment \\
\hline 128 & Piper thomsonii (C. DC.) Hook.f. & Piperaceae & Auni pan & $\mathrm{M}$ & $\mathrm{E}$ & Cough, cold; leaves chewed raw \\
\hline 129 & Polygonum microcephalum D. Don & Polygonaceae & Madhuxuleng & M & - & Dysentery \\
\hline 130 & Pteris ensiformisBurm. f. & Pteridaceae & - & $\mathrm{M}$ & $\mathrm{E}$ & Dysentery; tender frond edible \\
\hline 131 & Rhynchotechum ellipticum (Wall. ex D. Dietr.) A. DC. & Gesneriaceae & - & M & $\mathrm{E}$ & Cough, boils; edible \\
\hline 132 & Scoparia dulcis $\mathrm{L}$. & Plantaginaceae & Bondhania & M & - & Cough, bronchitis, kidney trouble \\
\hline 133 & Sida cordifolia $\mathrm{L}$. & Malvaceae & Hunborial & $\mathrm{M}$ & $\mathrm{E}$ & Post delivery trouble; yields fibre \\
\hline 134 & Smilax perfoliata Lour. & Smilacaceae & Baaghaasuralata & $\mathrm{M}$ & - & Wounds \\
\hline 135 & Solanum ferox $\mathrm{L}$. & Solanaceae & Katahibegena & M & - & Appetizer, antiasthmatic \\
\hline 136 & Spilanthes paniculata Wall. ex DC. & Asteraceae & Suhoni & M & - & Cough, toothache, constipation \\
\hline 137 & Stenochlaena palustris (Burm. f.) Bedd. & Blechnaceae & Dhekialata & M & $\mathrm{E}$ & $\begin{array}{l}\text { Fever, skin diseases; tender shoots edible, rhizomes } \\
\text { used in binding fish traps, anchor ropes, making } \\
\text { baskets }\end{array}$ \\
\hline
\end{tabular}




\begin{tabular}{|c|c|c|c|c|c|c|}
\hline 138 & Tetracera sarmentosa $(\mathrm{L}$.$) Vahl$ & Dilleniaceae & Oulata & - & $\mathrm{E}$ & $\begin{array}{l}\text { Leaves used as substitute for sand paper for } \\
\text { polishing, stem gives copious and potable water } \\
\text { when cut }\end{array}$ \\
\hline 139 & Tetrastigma thomsonianum Planch. & Vitaceae & Naltenga & - & $\mathrm{E}$ & Tender stem edible \\
\hline 140 & Tinospora sinensis (Lour.) Merr. & Menispermaceae & Hoguni lot & M & - & Gonorrhoea \\
\hline 141 & Uncaria sessilifructus Roxb. & Rubiaceae & Barakhialata & M & & Nervous disorder, hypertension \\
\hline 142 & Urena lobata $\mathrm{L}$. & Malvaceae & Hunborolua & M & $\mathrm{E}$ & $\begin{array}{l}\text { Diuretic, dysentery, rheumatism; } \\
\text { yields fibre, stem \& branches used as tooth brush }\end{array}$ \\
\hline 143 & Vitis planicaulis Hook. f. & Vitaceae & Panilata & M & - & Fever, sore throat \\
\hline 144 & Zingiber purpureum Rosc. & Zingiberaceae & Bon aada & M & - & Sprain, inflammation, paralysis \\
\hline \multicolumn{7}{|c|}{ Liana } \\
\hline 145 & Byttneria aspera Collebr. ex Wall. & Malvaceae & Tikonibarualata & M & $\mathrm{E}$ & Fever; young parts and bark used as hair wash \\
\hline 146 & Combretum roxburghii Spreng. & Combretaceae & Lotachali & - & $\mathrm{E}$ & Bark chewed with/as betel nut \\
\hline 147 & Connarus paniculatus Roxb. & Connaraceae & Makoilata & - & $\mathrm{E}$ & Oil (soap making) \\
\hline 148 & Dalbergia pinnata (Lour.) Prain & Papilionaceae & Laalengsali & - & $\mathrm{E}$ & Bark chewed with betel leaf \\
\hline 149 & Uvaria macrophylla Roxb. & Annonaceae & - & - & $\mathrm{E}$ & Ripe carpels edible \\
\hline \multicolumn{7}{|c|}{ Bamboo, cane and palm } \\
\hline 150 & Bambusa pallida Munro & Poaceae & Bijulibaah & - & $\mathrm{E}$ & $\begin{array}{l}\text { Edible young shoots, culms for making hut, baskets, } \\
\text { mats etc. }\end{array}$ \\
\hline 151 & Calamus erectus Roxb. & Arecaceae & Jeng bet & M & $\mathrm{E}$ & $\begin{array}{l}\text { Antidiabetic, antioxidant, dyspepsia; leaves for } \\
\text { roofing materials, cane, etc. }\end{array}$ \\
\hline 152 & Calamus flagellum Griff. ex Mart. & Arecaceae & Raaidang bet & - & $\mathrm{E}$ & Cane is used for various purposes \\
\hline 153 & Calamus floribundus var. depauperatus Becc. & Arecaceae & Lejaai bet & - & $\mathrm{E}$ & Fruits edible, stem for making basket \\
\hline 154 & Calamus tenuis Roxb. & Arecaceae & Jati bet & - & $\mathrm{E}$ & Cane is used for various purposes \\
\hline 155 & Pinanga gracilis $\mathrm{Bl}$. & Arecaceae & GerukaTamul & - & $\mathrm{E}$ & Fruits used as substitute of betel nut \\
\hline 156 & Pseudostachyum polymorphum Munro & Poaceae & Bojaalbaah & - & $\mathrm{E}$ & $\begin{array}{l}\text { The rhizomes are used for weaving sieves for } \\
\text { selecting young fish, the culms are split for weaving } \\
\text { fences, etc. }\end{array}$ \\
\hline 157 & Schizostachyum dullooa (Gamble) R. B. Majumder & Poaceae & Dolubaah & - & E & $\begin{array}{l}\text { Fencing, roofing, making baskets, mats, small boxes, } \\
\text { etc. }\end{array}$ \\
\hline
\end{tabular}


economic condition of the local inhabitants residing in and around the sanctuary. Local people utilized various plant resources in terms of medicine, timber and NTFP like, firewood, resin, gum, oil, dye, wild edibles, etc. The ethnobotanical importance of the various plant species enumerated in this sanctuary has been well documented by several workers (Kanjilal \& Bor 2005, Purkayastha et al. 2005, Purkayastha \& Nath 2006, Patiri \& Borah 2007, Das et al. 2008, Barbhuiya et al. 2009, Nath et al. 2010, Barukial \& Sarmah 2011, Sarkar \& Devi 2014b, Dutta et al. 2016) from different areas of the state. Plant species like Dillenia indica, Macaranga denticulata, Terminalia chebula, Clerodendrum infortunatum, Phlogacanthus thyrsiformis, Cheilocostus speciosus, Cissampelos pareira, Commelina benghalensis, Paederia scandens, Phyllanthus fraternus, etc. are well known to cure common health ailments such as fever, cough, cold, skin diseases and dysentery. Timber yielding species included trees such as Albizia lebbek, Dipterocarpus retusus, Magnolia hookeri, Premna bengalensis, etc. Few NTFP yielding species recorded from the study area includes Aquilaria malaccensis (resin/oil/highly scented wood), Canarium bengalense (resin), Morinda angustifolia (dye yielding stem and root), Melastoma malabathricum (stem and edible fruits), Diplazium esculentum (edible young and tender shoot), Connarus paniculatus (oil), Pinanga gracilis (edible fruits), Schizostachyum dullooa (bamboo), Calamus floribundus (cane), etc. A critically endangered and dominant tree of the study site (Sarkar \& Devi 2015), Vatica lanceaefolia, is a multipurpose plant species and was found to be collected frequently by the fringe people for fuel-wood. This critically endangered multipurpose tree species yields good quality firewood, resin as well as medicine. Calamus floribundus var. depauperatus is threatened to the northeastern region of India (Basu 1992) which is used for furniture. Artocarpus chaplasha and Ficus lepidosa are dominant food tree species of the H. hoolock inhabited in the sanctuary and contributed about 10-11\% to their annual diet (Borah 2016).

Anthropogenic activities like illegal felling (personal observation during study period) can exert disturbance to the survival and existence of these important species, and their structure and composition in the sanctuary. Illegal activities like tree felling have also been reported in the study area by other workers like Chakraborty \& Gupta (2005) and Sharma et al. (2010). If such interference continues or increases in future, it may pose a serious threat to the existence of the plant species diversity in this region. Nevertheless, quantification of the degree of disturbance exerted by biotic or abiotic factors is always necessary to understand the pressure and consequent effect on plant species richness and diversity of a particular area. Quantification of such parameters is also very essential for wise management of resources and to formulate conservation strategies accordingly.

\section{ACKNOWLEDGEMENTS}

Authors are grateful to the P.C.C.F (Wildlife) and D.F.O, Jorhat Division (Forest Department, Assam), for granting permission to carry out the work in Hollongapar Gibbon wildlife sanctuary. The kind assistance received from Deben Borah, other staff of Meleng Beat office and local residents during the field work is sincerely acknowledged. We also thank the staff of BSI, Arunachal Pradesh Circle, Itanagar for help and suggestions.

\section{REFERENCES}

Bajpai O, Pandey J \& Chaudhary LB (2016) Ethnomedicinal Uses of Tree Species by Tharu Tribes in the Himalayan Terai Region of India. Research Journal of Medicinal Plant 10(1): 19-41.

Barbhuiya AR, Sharma GD, Arunachalam A \& Deb S (2009) Diversity and conservation of medicinal plants in Barak valley, Northeast India. Indian Journal of Traditional Knowledge 8: 169-175.

Barukial J \& Sarmah JN (2011) Ethnomedicinal plants used by the people of Golaghat District, Assam, India. International Journal of Medicinal \& Aromatic Plants 1:203-211.

Basu SK (1992) Conservation status of Rattan in India. In: Basha SC \& Bhat KM (eds) Rattan Management and Utilization. KFRI, Kerala and IDRC, Canada, pp. 67-75.

Borah M \& Devi A (2014) Phenology, growth and survival of Vatica lanceaefolia B1.: A critically endangered tree species in a moist tropical forest of Northeast, India. Tropical Plant Research 1(3): 01-12.

Borah M. (2016) Feeding behaviour and analysis of food plant diversity of Hoolock hoolock in a Tropical Forest of Assam, India, Ph. D. thesis. Tezpur University, Tezpur, Assam, India, pp. 196.

Bujarbarua P \& Sarma SK (2006) A note on the diversity of family Poaceae in Gibbon Wildlife Sanctuary, Assam, India. Journal of Economic and Taxonomic Botany 30(1): 1-5.

Bujarbarua P (2002) An ecological study of Gibbon Wildlife Sanctuary, Jorhat, Assam, India, Ph. D. Thesis. Gauhati University, Guwahati, Assam, India.

www.tropicalplantresearch.com 
Chakraborty D \& Gupta AK (2005) Impact of habitat fragmentation on Hoolock Gibbon (Bunopithecus hoolock) in Gibbon Wildlife Sanctuary, Assam, India. In: Gupta AK (ed) Conservation of Hoolock Gibbon (Bunopithecus hoolock) in Northeast India. A Final Report of Wildlife Institute of India and United States Fish and Wildlife Services Collaborative Project, pp. 213-232.

Champion HG \& Seth SK (1968) A revised survey of the forest types of India. Government of India Press, New Delhi.

Chetia P \& Kalita DK (2012) Diversity and distribution of spiders from Gibbon Wildlife Sanctuary, Assam, India. Asian Journal of Conservation Biology 1(1): 5-15.

Chetry D (2001) Mammals of Gibbon Wildlife Sanctuary, Assam, India. Tiger Paper 28(4): 29-32.

Chetry D, Chetry R \& Bhattacharjee PC (2007) Hoolock: the ape of India. Gibbon Conservation Centre, Assam, pp. 23-26.

Das AK, Dutta BK \& Sharma GD (2008) Medicinal plants used by different tribes of Cachar district, Assam. Indian Journal of Traditional Knowledge 7: 446-454.

Dutta G, Baruah G \& Devi A (2016) Wild food plants of Mishing tribe- An ethnobotanical survey. Tropical Plant Research 3: 221-223.

Gadgil M (1989) Deforestation: Problems and Prospects. Foundation Day Lecture.Published in supplement to Wasteland News. Vol V No. 4. Society for Promotion of Wastelands Development, New Delhi, India.

Hazarika R \& Gupta AK (2005) Resource Sharing by Hoolock Gibbon (Bunopithecus hoolock) with two primate species in Gibbon Wildlife Sanctuary, Assam, India, Envis: Wildlife and Protected Areas.

Kanjilal UN \& Bor NL (2005) Flora of Assam. Omsons Publications, New Delhi, India.

Mehra A, Bajpai O \& Joshi H (2014) Diversity, utilization and sacred values of Ethno-medicinal plants of Kumaun Himalaya. Tropical Plant Research 1(3): 80-86.

Nath AJ, Raut A \& Bhattacharjee PP (2010) Traditional use of Barringtonia acutangula (L.) Gaertn. in fish farming in Chatla floodplain of Cachar, Assam. Indian Journal of Traditional Knowledge 9: 544-546.

Pandey D \& Pandey VC (2016) Sacred plants from ancient to modern era: Traditional worshipping towards plants conservation. Tropical Plant Research 3(1): 136-141.

Patiri B \& Borah A (2007) Wild edible plants of Assam. Director Forest Communication, Forest Department, Guwahati, Assam. Available from: http://assamforest.in/publication/wildEdible_plantsAssam.pdf (accessed 9 Sept. 2012).

PCCF (2012) Assam forest at a glance. Principal Chief Conservator of Forests \& Wildlife (PCCF), Forest Department, Guwahati, Assam.

Purkayastha J \& Nath SC (2006) Biological activities of ethnomedicinal claims of some plant species of Assam. Indian Journal of Traditional Knowledge 5: 229-236.

Purkayastha J, Nath SC \& Islam M (2005) Ethnobotany of medicinal plants from Dibru-Saikhowa Biosphere Reserve of Northeast India. Fitoterapia 76: 121-127.

Ramesh BR (2003) Biodiversity conservation and management. Tropical Ecology 44: 85-91.

Rodgers WA \& Panwar HS (1988) Planning a wildlife Protected Area Network in India. Department of Environment, Forests and Wildlife, Government of India, Wildlife Institute of India, Dehradun.

Sarkar M \& Devi A (2014a) Assessment of diversity, population structure and regeneration status of tree species in Hollongapar Gibbon Wildlife Sanctuary, Assam, Northeast India. Tropical Plant Research 1(2): 26-36.

Sarkar M \& Devi A (2014b). Tree species diversity of Gibbon Wildlife Sanctuary, Assam and its conservation perspectives. In: Biju Kumar A, Rajendran P \& Peethambaran CK (eds) Perspectives on Biodiversity of IndiaVol I. Centre for Innovation in Science and Social Action, Thiruvananthapuram, India, pp. 355-361.

Sarkar M \& Devi A (2015) Assessment of plant diversity in Hollongapar Gibbon Wildlife Sanctuary, Assam, Northeast India. Indian Forester 141(6): 648-659.

Sharma N, Madhusudan MD \& Sinha A (2010) Coexistence and conservation of seven sympatric primates in the fragmented habitat of Gibbon Wildlife Sanctuary, northeastern India. Available from: http://www.rufford.org/files/62.09.08\%20Detailed\%20Final\%20Report.pdf (accessed: 8 Aug. 2011).

Tilson RL (1979) On the behaviour of Hoolock gibbons (Hylobates hoolock) during different seasons in Assam, India. Journal of Bombay Natural History Society 76: 1-16.

Verma PK, Rawat KK \& Yadav A \& Das N (2012) The Liverwort and Hornwort flora of Hoollongapar Gibbon Sanctuary, Jorhat (Assam)-1. Archive for Bryology 152: 1-16. 